\title{
The phase-polarization curve of asteroid (3200) Phaethon
}

\author{
M. Devogèle ${ }^{1 \star}$ A. Cellino ${ }^{2}$, G. Borisov ${ }^{3,4}$, Ph. Bendjoya $^{5}$, J.-P. Rivet $^{5}$, L. Abe , $^{5}$ \\ S. Bagnulo ${ }^{3}$, A. Christou ${ }^{3}$, D. Vernet ${ }^{6}$, Z. Donchev ${ }^{4}$, I. Belskaya ${ }^{7}$, T. Bonev ${ }^{4}$, \\ and Yu. N. Krugly ${ }^{7}$ \\ ${ }^{1}$ Lowell Observatory, 1400 West Mars Hill Road, Flagstaff, AZ 86001 (U.S.A.) \\ ${ }^{2}$ INAF - Osservatorio Astrofisico di Torino, Pino Torinese, Italy \\ ${ }^{3}$ Armagh Observatory and Planetarium, College Hill, Armagh BT61 9DG, UK \\ ${ }^{4}$ Institute of Astronomy and National Astronomical Observatory, Bulgarian Academy of Sciences, 72, Tsarigradsko Chaussee Blvd., BG-1784 Sofia, Bulgaria \\ ${ }^{5}$ Université Côte d'Azur, Observatoire de la Côte d'Azur, CNRS, Laboratoire Lagrange UMR7293, Nice, France \\ ${ }^{6}$ Université Côte d'Azur, Obs. de la Côte d'Azur, UMR7293 CNRS Laboratoire Optique, Bv de l'Observatoire, CS 34229,06304 Nice, France \\ ${ }^{7}$ Institute of Astronomy of Kharkiv National University, Sumska Str. 35, Kharkiv 61022, Ukraine
}

\section{Accepted XXX. Received YYY; in original form ZZZ}

\begin{abstract}
A multi-colour phase-polarization curve of asteroid (3200) Phaethon has been obtained during the December 2017 apparition by merging measurements taken at the observing station of Calern (France) and at the Rhozen observatory (Bulgaria). All the observations were obtained in the positive polarization branch, the phase angle ranging from $36^{\circ}$ to $116^{\circ}$. The measured values of linear polarization are among the highest ever observed for a Solar system body. The covered interval of phase angle was not sufficiently extended to derive a firm determination of the $P_{\max }$ parameter, but this appears to occur at a phase angle around $130^{\circ}$ and reaches more than $45 \%$ of linear polarization. Phaethon is the parent body of the Geminid meteor shower, and the real physical nature of this object (asteroid or comet) has been a long-debated subject. Our polarimetric measurements seem to support the asteroid hypothesis with a phasepolarization curve similar to the asteroid (2) Pallas, but further observations at smaller phase angles are needed to draw definitive conclusions.
\end{abstract}

Key words: - minor planets, asteroids: individual: (3200) Phaethon - meteors - techniques: polarimetric

\section{INTRODUCTION}

The near-Earth asteroid (NEA) (3200) Phaethon is a very interesting object in many respects. It has an estimated size of about $5 \mathrm{~km}$ (Hanuš et al. 2016). Its most outstanding properties are:

- A very large orbital eccentricity, that produces a very small perihelion distance.

- The fact of being a member of an unusual taxonomic class of low-albedo objects.

- The fact of being the most likely parent body of the Geminid meteor shower.

The osculating orbital elements of Phaethon, taken from the NeoDys web site ${ }^{1}$ are listed in Table 1 . They indicate that this object dynamically belongs to the Apollo orbital class of the NEA

\footnotetext{
* Partly based on data collected with 2-m RCC telescope at Rozhen National Astronomical Observatory, Bulgaria

${ }^{1} \mathrm{http}: / /$ newton.dm.unipi.it/neodys $2 /$
}

Table 1. Osculating elements of (3200) Phaethon at epoch 58200.0 MJD

\begin{tabular}{lccc}
\hline & Value & $1-\sigma$ variation & Units \\
\hline$a$ & 1.27135 & $3.412 \mathrm{e}-10$ & $\mathrm{AU}$ \\
$e$ & 0.889933 & $6.935 \mathrm{e}-09$ & \\
$i$ & 22.256 & $2.491 \mathrm{e}-06$ & $\mathrm{deg}$ \\
$\Omega$ & 265.226 & $2.038 \mathrm{e}-06$ & $\mathrm{deg}$ \\
$\omega$ & 322.173 & $2.108 \mathrm{e}-06$ & $\mathrm{deg}$ \\
$M$ & 38.954 & $8.531 \mathrm{e}-07$ & $\mathrm{deg}$ \\
\hline
\end{tabular}

population. During their orbital motion, these objects sweep regions inside the orbit of the Earth, but what makes Phaethon a quite peculiar Apollo is its perihelion distance $q=0.1399 \mathrm{AU}$, one of the smallest values ever found for an asteroid, and a consequence of its very high orbital eccentricity of about 0.89 .

In terms of taxonomy, Phaethon was originally classified by Tholen (1985) as a member of the F class. This class, first introduced by Gradie and Tedesco (1982), included asteroids characterized by an overall lack of absorption features, and exhibiting a flat (F-class) reflectance spectrum down to the very blue region, 
where other classes tend to display some significant absorption bands. Based on IRAS thermal IR data, Tedesco et al. (2002) found the albedo of Phaethon to be 0.11 which is higher than the typical value for the F-class which ranges between 0.03-0.07. In the case of Phaethon, recent estimates based on joint modeling of IRAS and Spitzer data gives $0.12+/-0.01$ Hanuš et al. (2016). In the Tholen (1984) taxonomy, only 27 asteroids (3\% of all classified objects) were found to belong to this class. F-class asteroids are therefore uncommon. Based on their properties, Gaffey et al. (1989) suggested that they are most probably primitive objects, having reflectance spectra similar to those of CI1 - CM2 meteorite assemblages, and might likely include some organic compounds on their surfaces.

Recently, however, the $\mathrm{F}$ taxonomic class has been removed from taxonomic classifications based on spectroscopic observations using modern CCD devices (Bus and Binzel 2002; DeMeo et al. 2009), because the blue part of the reflectance spectrum is no longer adequately covered. Objects previously classified as $\mathrm{F}$ have now been merged together with old B-class asteroids, which were previously distinct, in a unique class named $\mathrm{B}$, which includes objects having featureless reflectance spectra, flat or slightly blueish in the wavelength interval between about 0.5 and $1 \mu \mathrm{m}$.

Since the epoch of its discovery in 1983, based on data collected by the IRAS satellite, (3200) Phaethon has been considered to be the long sought parent body of the Geminid meteor shower, since its orbit was found to be practically coincident with that of 20 Geminid fireballs photographed in the 50s by the Prairie Network cameras. (see, eg Gustafson (1989) and references therein). The origin of several Geminid fireballs from Phaethon were also found by this author to be consistent with episodes of cometary activity of their assumed parent body.

Based on all the above mentioned properties, a natural question arises: is Phaethon an asteroid or a comet? In this paper, we use the results of our recent polarimetric measurements of this object to provide some additional arguments to considered in this discussion. Though we can say that our new data are not yet sufficient to draw definitive conclusions, and waiting for some new data that we plan to obtain later this year during the next apparition of Phaethon, we already see evidence supporting one of the two alternative hypotheses concerning the real nature of this object.

\section{2 (3200) PHAETHON: ASTEROID OR COMET?}

The following arguments can be used to support or contradict the two hypotheses about the real nature of Phaethon.

First of all, it is commonly accepted that the NEA population includes also objects which were originally born as comets, and achieved their current orbits after a complex dynamical evolution. A recent estimate of the relative abundance of cometary bodies among NEAs is $8 \% \pm 5 \%$ (DeMeo and Binzel 2008).

Moreover, in recent years, the discovery of so-called mainbelt comets, objects with typically asteroidal orbits, but found to exhibit transient cometary activity, has clearly shown that the classical distinction between asteroids and comets is probably not as sharp as we were led to believe in the past (Hsieh and Jewitt 2006). In this respect, Jewitt and Hsieh (2006) presented observations of (155140) 2005 UD, an another small Apollo asteroid. Based on its orbital and spectroscopic similarities with Phaethon, taking into account also the different sizes of the two objects (2005 UD being only about $1 \mathrm{~km}$ in size) reached the conclusion that both Phaethon and 2005 UD might be separate pieces of a unique main-belt comet parent body that suffered disruption after reaching a Sun-approaching orbit.

It is important to note that, among objects originally classified as members of the old F-class, there is at least one major example of a body, (4015) Wilson-Harrington (see Bowell et al. (1992)) that was originally considered to be an F-class asteroid, but was later found to exhibit cometary activity. No further activity has been detected again in recent years for Wilson-Harrington, suggesting that this object is comet very close to becoming extinct (Fernández et al. 2005). Based on the observed $\mathrm{CN}$ spectral band emission at $0.388 \mu \mathrm{m}$, which is typical of cometary activity, Chamberline et al. (1996) suggested that (3200) Phaethon could be another inactive cometary remnant.

The possibility that asteroids originally classified as members of the old F-class display properties diagnostic of a cometary origin is exciting. A major point is that the old F-class asteroids can still be distinguished today based on their uncommon polarimetric properties. In particular, the phase-polarization curves of these bodies are characterized by unusually low values, of about $17^{\circ}$ according to Belskaya et al. (2017), of the so-called polarimetric inversion angle, that is the value of phase angle at the point of transition from the negative polarization branch to the positive polarization branch. In other words, the inversion angle marks the transition from the interval of phase angles for which the measured plane of linear polarization is parallel to the Sun-target-observer plane (the scattering plane) to the interval of phase angles for which the plane of linear polarization becomes normal to the scattering plane (Belskaya et al. 2005; Cellino et al. 2015a; Belskaya et al. 2017). For "regular" asteroids, the value of the inversion angle is larger than $18^{\circ}$ and in the vast majority of cases it is around $20^{\circ}$ or larger (Belskaya et al. 2005; Cellino et al. 2016). Interestingly, polarimetric observations of two comets, 2P/Encke (Boehnhardt et al. 2008) and 133P/ElstPizarro (Bagnulo et al. 2010) showed that their nuclei exhibit a polarimetric behavior characterized by low inversion angles, similar to those of known F-class asteroids. This supports previous suggestions Kolokolova and Jockers (1997) that a low inversion angle of polarization could be diagnostic of a cometary surface.

These are the main reasons why we decided to perform a campaign of polarimetric observations during the last apparition of Phaethon.The simple idea was to obtain a phase-polarization curve, to be compared with those of typical F-class asteroids, to check whether they share the same properties, in support a likely cometary origin of Phaethon. These observations, described in Section 3, are discussed in Section 4. This apparition was also a unique opportunity to obtain measurement at a wide range of phase angle which allow to extend the phase coverage for this type of object and to compare them with either main belt asteroid measurements at lower phase angle and other NEOs.

On the other hand, there are arguments suggesting that (3200) Phaethon could be more likely an asteroid, rather than a comet. First of all, the very belonging of Phaethon to the F class is not completely certain. Although Tholen (1985) assigned Phaethon to the F class based on a clear absence of an UV absorption feature, Cochran and Barker (1984) had previously reported a reflectance spectrum of Phaethon exhibiting a definite UV absorption feature.

Later, Halliday (1988) pointed out that observations of Geminid fireballs suggested bulk densities between 0.7 and $1.3 \mathrm{~g} / \mathrm{cm}^{3}$, lower than the typical densities of meteorites, but higher than the values generally inferred for fireballs. This would suggest that Phaethon is not a (typical) comet. In this respect, it is important to note also that De León et al. (2010), based on a comparison of the reflectance spectra, suggested that (3200) Phaethon could be a 
fugitive member of a dynamical family whose parent body is the large main belt asteroid (2) Pallas. These authors noted that dynamical evolutionary paths possibly linking Phaethon with Pallas do exist. However, the spectrum of Phaethon is generally bluer than that of Pallas. The spectral similarity seems to be stronger between Phaethon and some small members of the Pallas family, something that the above authors generically explained as a consequence of size-dependent surface effects. Finally, these authors also found that no other asteroid belonging to the modern $\mathrm{B}$ taxonomic class exhibits a comparable spectral similarity.

Finally, Hanuš et al. (2016) derived, using a sophisticated thermophysical model, a new value of $0.12 \pm 0.01$ for the geometric albedo of Phaethon. This value is much higher than previous estimates based on thermal IR IRAS data, and may be too high for a typical comet (just for a comparison, the geometric albedo derived for comet 67P/Churyumov-Gerasimenko from Rosetta observations is $0.062 \pm 0.02$, according to Ciarniello et al. (2015)). A value of 0.12 for the geometric albedo is rather high also for lowalbedo asteroids belonging to both the old F-class and the modern B-class. We note, however, that, in this respect, (2) Pallas has also a relatively high albedo value of 0.145 according to Shevchenko and Tedesco (2006). Such value, which is anomalous when looking at the relations between geometric albedo and polarimetric properties (Cellino et al. 2015b) is confirmed, however, by independent thermal radiometry estimates (Masiero at al. 2011).

In this situation, we carried out our campaign of polarimetric observations of Phaethon.

\section{THE OBSERVATIONS}

The observations were done at the Calern observing station (MPC 010) of the Observatoire de la Côte d'Azur (France) and at the Bulgarian National Astronomical Observatory (BNAO), Rozhen (MPC 071), Bulgaria. At Calern, the Omicron (west) telescope of the C2PU facility was used with the Torino Polarimeter (ToPol) mounted on its Cassegrain focus (F/12.5). ToPol is a WedgedDouble Wollaston polarimeter (Oliva 1997). Its optical configuration makes it possible to obtain simultaneously four images corresponding to rotations of the linear polarization plane of $0^{\circ}, 45^{\circ}, 90^{\circ}$ and $135^{\circ}$, from which the $Q$ and $U$ Stokes parameters can be measured in one single exposure. ToPol is described in Pernechele at al (2012). A description of the instrument and the reduction techniques used for this instrument have also been described by Devogèle et al (2017).

Polarimetric observations at BNAO-Rozhen were performed using the 2-Channel-Focal-Reducer Rozhen (FoReRo2) (Jockers et al. 2000) attached at the Cassegrain focus of the $2 \mathrm{~m}$ RitcheyChrétien-Coudé (RCC) telescope. In polarimetric mode, FoReRo2 is equipped with a Wollaston prism, placed in the parallel beam of the instrument. Recently, the instrument was equipped with a retarder half-wave-plate. This makes it possible to easily rotate the light electric vector before the Wollaston prism by steps of $45^{\circ}$, (by simply rotating the retarder by steps of $22.5^{\circ}$ ), and to use the so called "beam swapping technique", described in Bagnulo et al. (2009), to measure accurately the linear polarization of the observed source.

At Calern, the polarimetric observations were performed in four colours, using standard BVRI filters, as long as the target was sufficiently bright and high above the horizon to allow for multicolour measurements. We remind that Phaethon was moving at a very fast rate, with its apparent brightness and elevation decreasing from night to night, as the phase angle was steadily increasing. This means that during the last nights when it was still observable, we could only perform observations in $\mathrm{V}$ and $\mathrm{R}$ colours, which require shorter exposure times. At Rozhen, all measurements were done in $\mathrm{R}$ light, only.

\section{DISCUSSION}

Figures 1 and 2 show the results of our polarimetric campaign. The corresponding data are listed in Table A1. In the above Figures the nominal error bars of the measurements are shown, and they are often non-negligible, as shown in Table A1, but due to the large interval covered by the polarization measurements, the error bars are hardly visible in the plot. The interval of covered phase angle is large, between $36^{\circ}$ and $116^{\circ}$. Very high values of linear polarization have been measured, steadily increasing with the phase angle, although at the highest values it seems that the phase-polarization curve tends to become shallower, as expected for an object approaching $P_{\max }$, the value of maximum positive polarization. We note that, as shown by the right Panel of the same Figure, the Calern and Rozhen measurements, obtained independently using two different instruments, agree with each other in the R colour, and at the same time the $\mathrm{V}$ and $\mathrm{R}$ measurements also agree closely. The same is true for all the multi-colour measurements shown in Fig. 2. It is important to note that there is a regular pattern in the measurements obtained in different colours. In particular, although this is not very clear in Fig. 2 because of the scale of the polarization axis, the measured linear polarization exhibits a slight decrease for decreasing wavelength, from I to B. This follows a pattern which is known to characterize the positive polarization branch of lowalbedo asteroids (Belskaya et al. 2005; Bagnulo et al. 2014).

In order to obtain estimates of some of the relevant polarimetric parameters, we computed a best-fit curve of the available phasepolarization data in $\mathrm{V}$, using the trigonometric representation of the phase-polarization relationship of asteroids originally proposed by Lumme and Muinonen (1993), and lately adopted by other authors, including, for instance, Penttila at al. (2005):

$P_{\mathrm{r}}(\alpha)=A \sin ^{B}(\alpha) \cdot \cos ^{C}\left(\frac{\alpha}{2}\right) \cdot \sin \left(\alpha-\alpha_{i n v}\right)$

where $P_{\mathrm{r}}(\alpha)$ is the fraction of linear polarization (with a sign which is negative in the negative polarization branch, see, for instance, Cellino et al. (2015b)), $\alpha$ is the phase angle, $\alpha_{i n v}$ is the value of the inversion angle of polarization, and $A, B$ and $C$ are coefficients to be computed by means of least-squares fits. This mathematical representation of the phase-polarization curves of atmosphereless Solar system bodies is eminently empirical (Muinonen, private communication). It was found to provide very reasonable fits of the phase-polarization curves of objects observed over wide intervals of phase angle, much wider than those possible for main belt asteroids (which cannot be observed at phase angles much larger than about $30^{\circ}$ ).

The best-fit representation of our $\mathrm{V}$ data is shown in the left Panel of Fig. 1. It can be seen that the computed curve fits very well the available data, including, in addition to our Calern data, also one single measurement available in the literature, obtained at a phase angle of $23^{\circ}$ by Fornasier et al. (2006). The computed chisquare value of the fit is 0.19 . Some noise can also be due to a mild periodic variation of the linear polarization, that we have found to be synchronous with the rotation period and therefore most likely due to some surface heterogeneity. This is the subject of a separate 

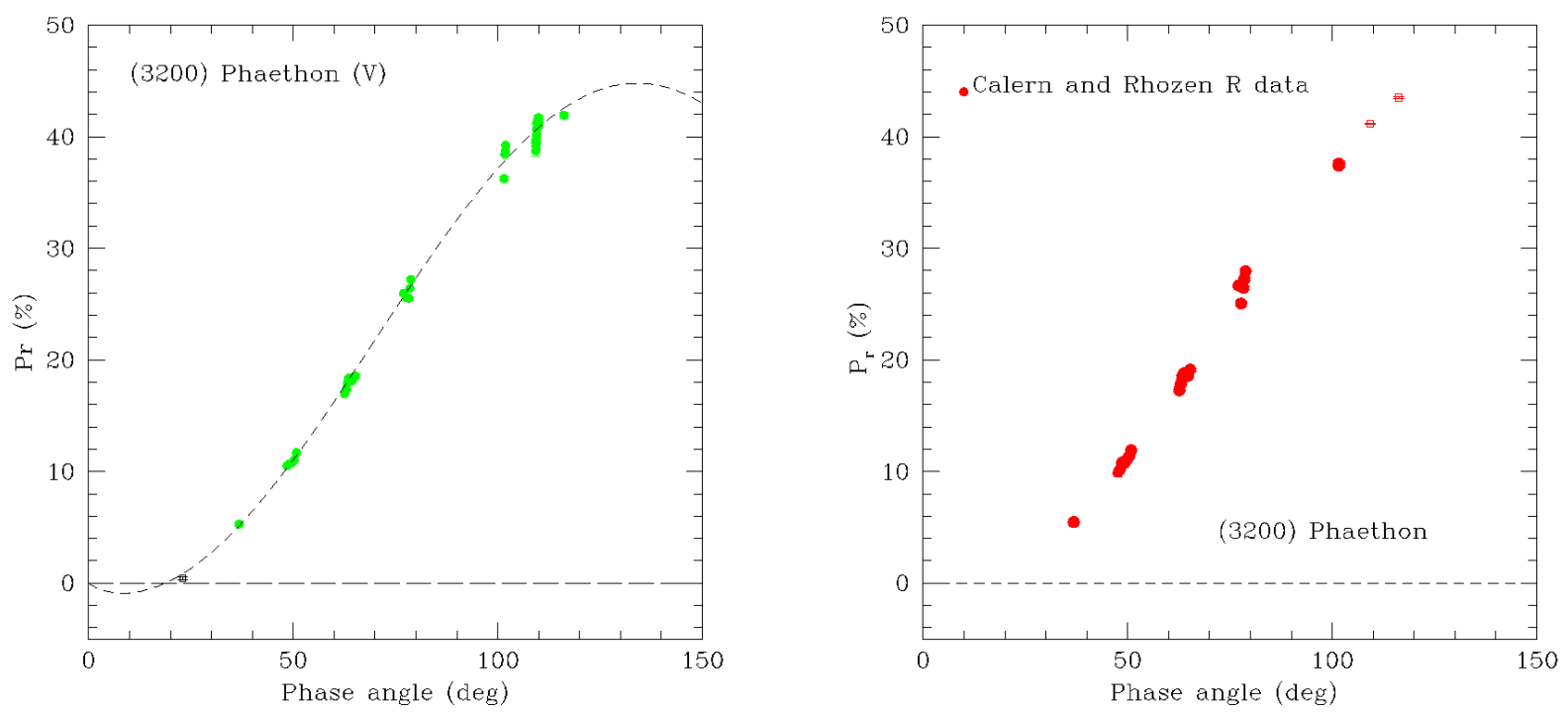

Figure 1. Left panel: Phase-polarization curve of (3200) Phaethon in V light. New Calern data (green symbols) plus one data point (black square) previously available in the literature. The best-fit curve computed using the trigonometric representation (see text) is also shown. Right Panel: Phase-polarization of (3200) Phaethon in R light. Calern and Rozhen data are merged together. Rozhen measurements are indicated by empty symbols, which are visible only at the highest values of phase angle, where they do not overlap with simultaneous measurements done at Calern.

paper (Borisov et al., in preparation). The value of the resulting inversion angle and the rms residuals for the whole data set are given in Table 2. By looking at Fig. 1, a few considerations can be immediately done: first, although the best-fit model for the $\mathrm{V}$ data using the trigonometric representation is quite good, the range of phase angles covered by available measurements is not sufficient to allow us to obtain a very robust determination of the value $P_{\max }$ of the maximum positive polarization. On the other hand, if the adopted representation of the phase-polarization curve is valid also slightly beyond the maximum value of phase angle covered by our data, then we find that the value of $P_{\max }$ should be reached at a phase angle of about $130^{\circ}$, and is about $45 \%$, a very high value, much higher than the $P_{\max }$ values determined so far for any of Solar system body for which polarimetric measurements were made.

The second main consideration concerns the derived value of the inversion angle $\alpha_{i n v}$. According to our computations, this value is about $19^{\circ}$ for the whole set of Phaethon V measurements. Such a value is hardly consistent with the $\mathrm{F}$ taxonomic class. On the other hand, the main uncertainty affecting this estimate is due to the fact that our data do not cover the negative polarization branch, and is therefore based on an extrapolation to smaller values of phase angle of measurements covering the positive polarization branch, only.

Since we seek evidence in favour of, or against, the possibility that Phaethon belongs to the $\mathrm{F}$ taxonomic class, based on its polarimetric properties, and in particular on the value of its polarization inversion angle, we computed trigonometric fits of sets of measurements obtained by merging together the Phaethon polarization data with those of a number of asteroids belonging to the modern Bclass defined by Bus and Binzel (2002), including also some of the most important members of the old F-class, characterized by inversion angles of polarization generally between $16^{\circ}$ and $17^{\circ}$, significantly smaller than those of the B-class. In particular, we considered asteroids (2) Pallas, (24) Themis, (47) Aglaja, (59) Elpis, as representatives of non-F objects belonging to the modern B-class, as well as some members of the old F-class, namely (142) Polana, (213) Lilaea, (419) Aurelia, (762) Pulkova, and (704) Interamnia.
Table 2. Results of the best-fit of available phase-polarimetric data obtained in V light for (3200) Phaethon alone, or for a combination of data for Phaethon and other known B-class asteroids, some of them having been classified in the past as members of the old F-class.

\begin{tabular}{lrcc}
\hline Data set & $\begin{array}{c}\text { Number of } \\
\text { measurements }\end{array}$ & $\begin{array}{c}\text { Inversion angle } \\
(\mathrm{deg})\end{array}$ & $\begin{array}{c}\text { rms } \\
(\%)\end{array}$ \\
\hline$(3200)$ alone & 45 & $18.8 \pm 1.6$ & 0.715 \\
$(3200)+(2)$ & 73 & $19.2 \pm 0.3$ & 0.606 \\
$(3200)+(24)$ & 57 & $19.9 \pm 0.6$ & 0.658 \\
$(3200)+(47)$ & 58 & $19.2 \pm 0.5$ & 0.652 \\
$(3200)+(59)$ & 55 & $19.4 \pm 0.4$ & 0.655 \\
$(3200)+(142)$ & 47 & $17.8 \pm 1.2$ & 0.699 \\
$(3200)+(213)$ & 52 & $19.3 \pm 0.5$ & 0.715 \\
$(3200)+(419)$ & 57 & $19.0 \pm 0.5$ & 0.700 \\
$(3200)+(704)$ & 83 & $16.8 \pm 0.5$ & 0.769 \\
$(3200)+(762)$ & 51 & $18.5 \pm 0.8$ & 0.676 \\
$(3200)+(142),(213)$, & 110 & $17.0 \pm 0.4$ & 0.690 \\
$(419),(704),(762)$ & & & \\
$(213)+(704)$ & 45 & $16.2 \pm 0.1$ & 0.241 \\
\hline
\end{tabular}

All the above asteroids are classified as B-type by Bus and Binzel (2002), with the two exceptions of (419) and (762), for which there is no Bus and Binzel (2002) classification. We note also that, in the most recent taxonomic classification by DeMeo et al. (2009), both (2) Pallas and (3200) Phaethon are classified as members of the modern B-class, whereas (24) Themis is classified as C-type. It is interesting to note that (762) Pulkova is a known binary asteroid, formed by a primary component being about $140 \mathrm{~km}$ in size, and a much smaller satellite. Finally, we note that the above list of objects includes asteroids of quite different sizes. The biggest ones being (2) Pallas, (24) Themis and (704) Interamnia, with diameters above 500,200 and $300 \mathrm{~km}$, respectively, while some other objects, like (142) Polana, are smaller than $60 \mathrm{~km}$ (Masiero at al. 2011).

For all selected asteroids we used all polarimetric measure- 


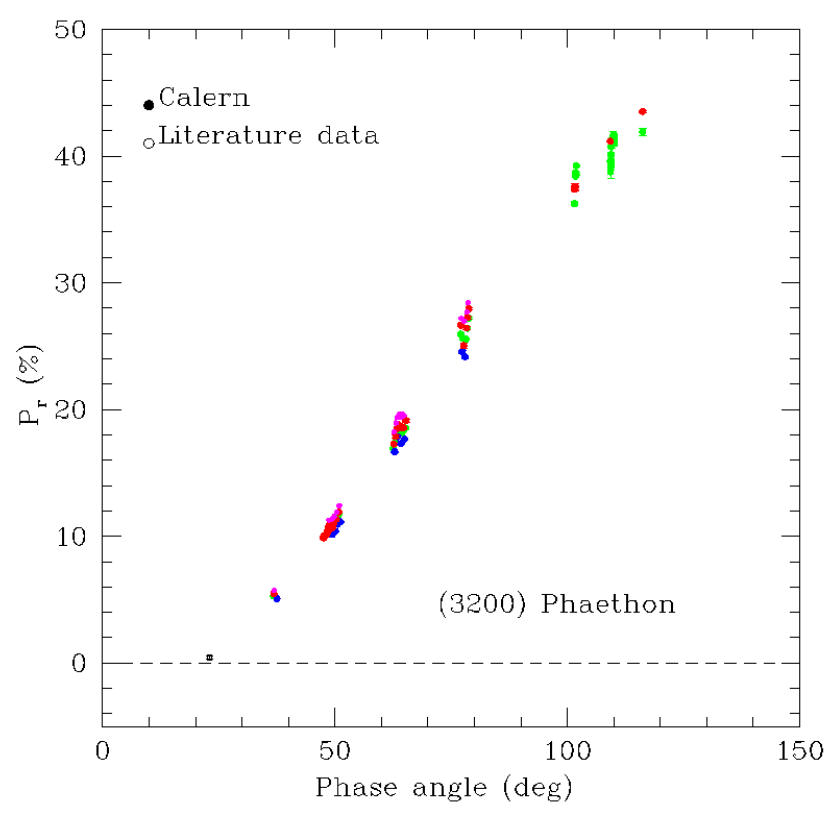

Figure 2. Calern and Rozhen polarimetric data for (3200) Phaethon in BVRI filters. The corresponding symbol colours are blue, green, red and magenta, respectively. The single data point taken from the literature in Vcolour is shown as a black point. Points corresponding to different colours tend to overlap in this plot, but there is a regular trend of increasing positive polarization going from B to I, as can be seen looking at the data shown in Table A1. Only V and R data were obtained at the highest phase angles, when the object was becoming fainter and located at small elevation above the horizon.

ments available in the literature ${ }^{2}$, plus a few still unpublished data obtained at Calern in the the framework of the Calern Asteroid Polarimetric Survey (CAPS). These measurements will be included in a new CAPS data release to be published in a paper currently in preparation.

The results of our exercise are listed in Table 2 and graphically shown in Figs. 3-6. Fig. 3 shows what happens when we add to our set of Phaethon measurements in $\mathrm{V}$ just a couple of data points covering the negative polarization branch. In particular, we use here the only two measurements obtained in the past at the CASLEO observatory (Argentina) for (142) Polana, an asteroid belonging to the old F-class. The figure shows that, obviously, the best-fit curve is mostly constrained by the large number of Phaethon measurements, covering a very large interval of phase angles. However, as shown in Table 2, adding the two Polana data points produces a shift of the resulting inversion angle of polarization of about one degree towards smaller values, from $18.8^{\circ}$ to $17.8^{\circ}$, producing at the same time a negligible improvement of the rms. An inversion angle of $17.8^{\circ}$, however, does not constitute strong evidence that Phaethon is a member of the F-class, because, according to Belskaya et al. (2017), one should expect slightly smaller values for the inversion angle of this class.

It is therefore interesting to compute the best-fit curve using

2 The data come mostly from the Planetary Data System repository, available at the URL address http://pds.jpl.nasa.gov/ (files maintained by D.F. Lupishko and I.N. Belskaya), and from some recently published articles, including (Gil-Hutton et al. 2014; Belskaya et al. 2017; Devogèle et al 2017) the same trigonometric representation, in cases when one adds to the Phaethon data larger numbers of measurements of F-class asteroids, in order to see the change in the fitted inversion angle of polarization and in terms of rms residuals. The results of this exercise are shown, again, in Table 2, and graphically in Fig. 4 for each of the F-class asteroids (213), (419), (704) and (762), and in Fig. 5, in which the data of all the above-mentioned F-class asteroids are merged together.

By looking at these plots and at the corresponding values obtained in the different cases for the inversion angle and the rms residuals, it seems that the hypothesis that Phaethon can be a member of the F-class is not strongly supported by the data. The resulting curves show that the measurements of F-class asteroids are not particularly well fitted. This is evident for objects like (213), (419) and (704), for which fairly large numbers of measurements are available. The obtained rms residuals confirm this conclusion. In terms of inversion angle, the obtained values are also larger than expected if Phaethon was an F-class asteroid, characterized by a phase-polarization curve fully compatible with those of other objects of the same class. Only by adding to the Phaethon data all the available data of the five F-class objects mentioned above, the resulting best-fit curve reaches an inversion angle of $17^{\circ}$, but the rms do not improve and a visual inspection of the results, shown in Fig. 5, does not provide convincing evidence that the obtained bestfit is particularly good, although we cannot firmly state that the data of Phaethon are clearly incompatible with those of the F-class. It is clear that, in order to draw definitive conclusions, we need to observe Phaethon when it is visible at phase angles corresponding to the negative polarization branch.

On the other hand, if one repeats the same exercise, but merging this time our Phaethon data with available data for modern Bclass but non-F-class, asteroids, the situation seems to change. In particular, as shown in Table 2 and in Fig. 6, that available polarimetric data for (24) Themis and (47) Aglaja are clearly not compatible with the phase-polarization curve of Phaethon. The situation is better in the case of (59) Elpis. The best result is obtained, however, in the case of the data of (2) Pallas. By merging Phaethon and Pallas data together, the rms of the resulting best-fit curve improves significantly, and this is also clearly visible by looking at the top-left Panel of Fig. 6. The corresponding inversion angle of polarization turns out to be $19.2^{\circ} \pm 0.3^{\circ}$, a value fully compatible with the one found for the data of Phaethon alone, but with a smaller associated uncertainty.

Our observations allow to estimate polarimetric albedo based on the empirical relationships "polarimetric slope-albedo" and "Pmax-albedo" (e.g., Geake and Dollfus (1986)). In both cases the polarimetric albedo of Phaethon is estimated to be very low (about $5 \%$ ). This value is quite different from the determined radiometric albedo of Phaethon Hanuš et al. (2016). What is the possible reason of such discrepancy? First of all, a thorough check of Phaethon's albedo determined from the radiometric data is required. If there are any doubts in rather high surface albedo of Phaethon we should assume that the polarimetric method of albedo determination failed in the case of this asteroid. This could happen if the surface texture of Phaethon is very different from other asteroids for which polarimetric measurements are available, According to laboratory measurements $P_{\max }$ depends on the grain size and increases for dustfree surfaces (e.g. Geake and Dollfus (1986)). However, an absence of regolith layer seems to contradict thermophysical modeling. 

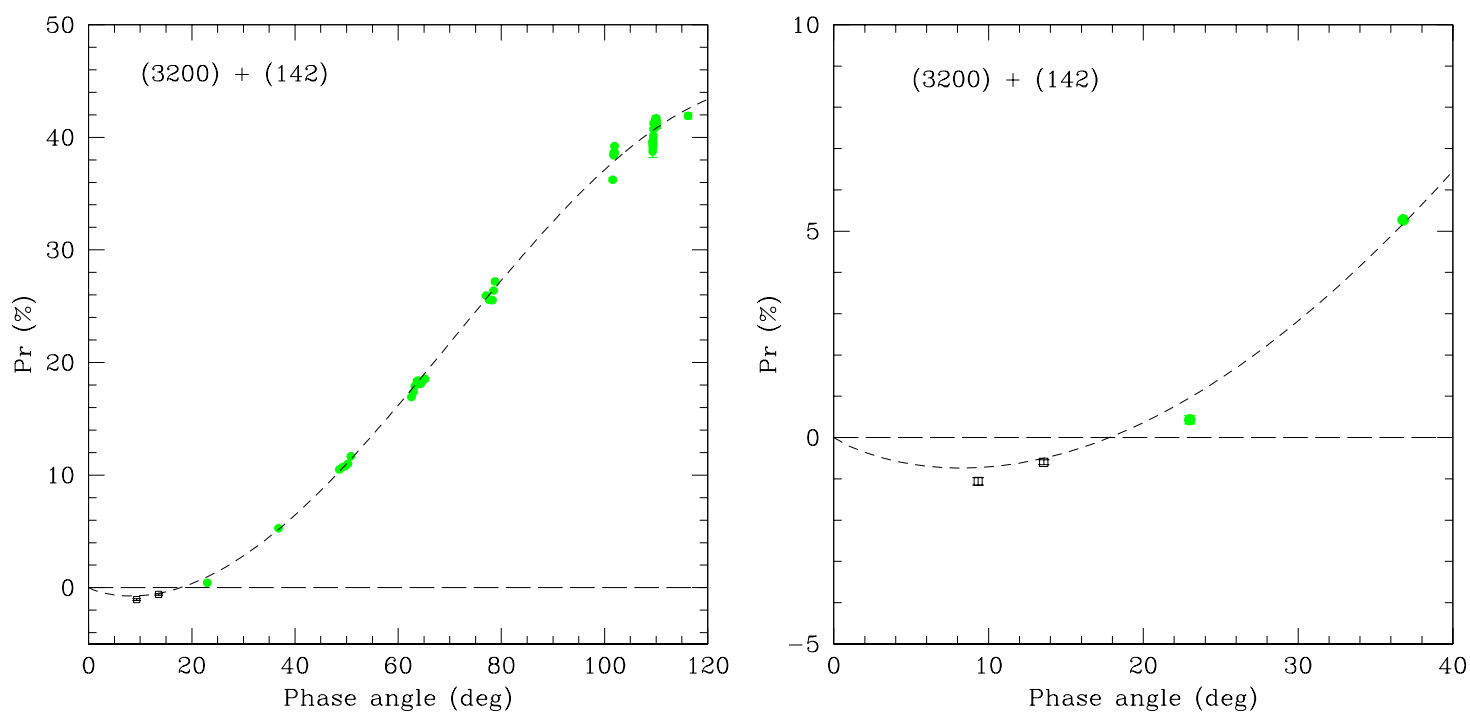

Figure 3. Best-fit phase-polarization curve of the data set obtained by adding to available Phaethon polarization measurements in V light just the two measurements available for the F-class asteroid (142) Polana, both covering the negative polarization branch. The left Panel shows the full data and the corresponding best-fit. For sake of clarity, the right Panel covers only the region of phase angle $<40^{\circ}$, where only two measurements are available for (3200) Phaethon (displayed in green).

\section{CONCLUSIONS}

Although not yet sufficient to derive conclusive evidence about the value of the inversion angle of (3200) Phaethon, which is a decisive factor in assigning it to the F-class, a characterization that could be considered to strongly support the hypothesis of a cometary origin, the measurements presented in this paper seem rather to suggest that Phaethon does not belong to the F taxonomic class. We plan to confirm this conclusion by means of further polarimetric measurements that we hope to obtain next December during the next apparition of this object, when it will be observable at phase angles within the negative polarization branch.

For the moment, we can state that, by combining our new polarimetric measurements of Phaethon with those available for main belt asteroids which are members of the modern B class, we find that the best agreement is found when we merge Phaethon polarimetric data with those of (2) Pallas. This seems to confirm the results of previous analyses, in particular those presented by De León et al. (2010), reported in Section 2, which suggested that Phaethon could be a fugitive member of the Pallas dynamical family, presumably produced by a big cratering event on this very large asteroid (Milani at al. 2014)

If this conclusion is correct, and confirmed by future data, the problem of the origin of Phaethon can be considered solved. On the other hand, other questions remain open, including the following:

- What is the real composition of Pallas and its siblings, taking into account that the geometric albedo of Pallas (Cellino et al. 2015b), and also of Phaethon, according to Hanuš et al. (2016), is so high with respect to other asteroids belonging to the $\mathrm{B}$ taxonomic class?

- Is Pallas rich in volatiles, that can be found also in its siblings, possibly producing episodes of cometary activity? Is the Pallas family a good candidate to look for new main-belt comets?

- What is the origin of Pallas? Was it accreted in its present location, or was it injected into the asteroid main belt coming from regions at larger heliocentric distance?

- The reflectance spectrum of Phaethon is very similar to those of other small members of the Pallas family (De León et al. 2010) which do not reach the extremely small perihelion distance as Phaethon, and are not subject to close encounters with the inner planets. Has this some consequence about current ideas of the effects of space weathering and resurfacing due to tidal effects produced by close encounters with the inner planets?

Of course, another possible open question concerns the polarimetric properties of the old $\mathrm{F}$ taxonomic class. In particular, is it possible that this class might be characterized by a larger interval of the inversion angle $\alpha_{i n v}$ than previously believed? In some cases this might make it hard to distinguish them from the similar B-class based on polarimetric data alone. Fig. 7 shows that for the case of (213) Lilaea and (704) Interamnia, some difference between the phase-polarization curves may exist, but in any case the inversion angle is small for both objects. The possibility of a wider heterogeneity in the polarimetric properties of the F-class would raise some doubts about the predictive power of polarization measurements. This potential problem can be assessed only by means of a survey of spectroscopic measurements extending into the blue region of the reflectance spectrum for objects found to be characterized by a small value of the inversion angle of polarization, in order to associate more closely the polarimetric and spectral reflectance properties of the F-class. Hopefully, this is something that will be achieved by examining the next data releases of Gaia observations of Solar system objects, when spectroscopic data, including also the blue region of the reflectance spectrum, will be included for progressively increasing numbers of asteroids. In the mean time, we will continue our Calern Asteroid Polarimetric Survey program, and will increase the data set of asteroid polarimetric data. 

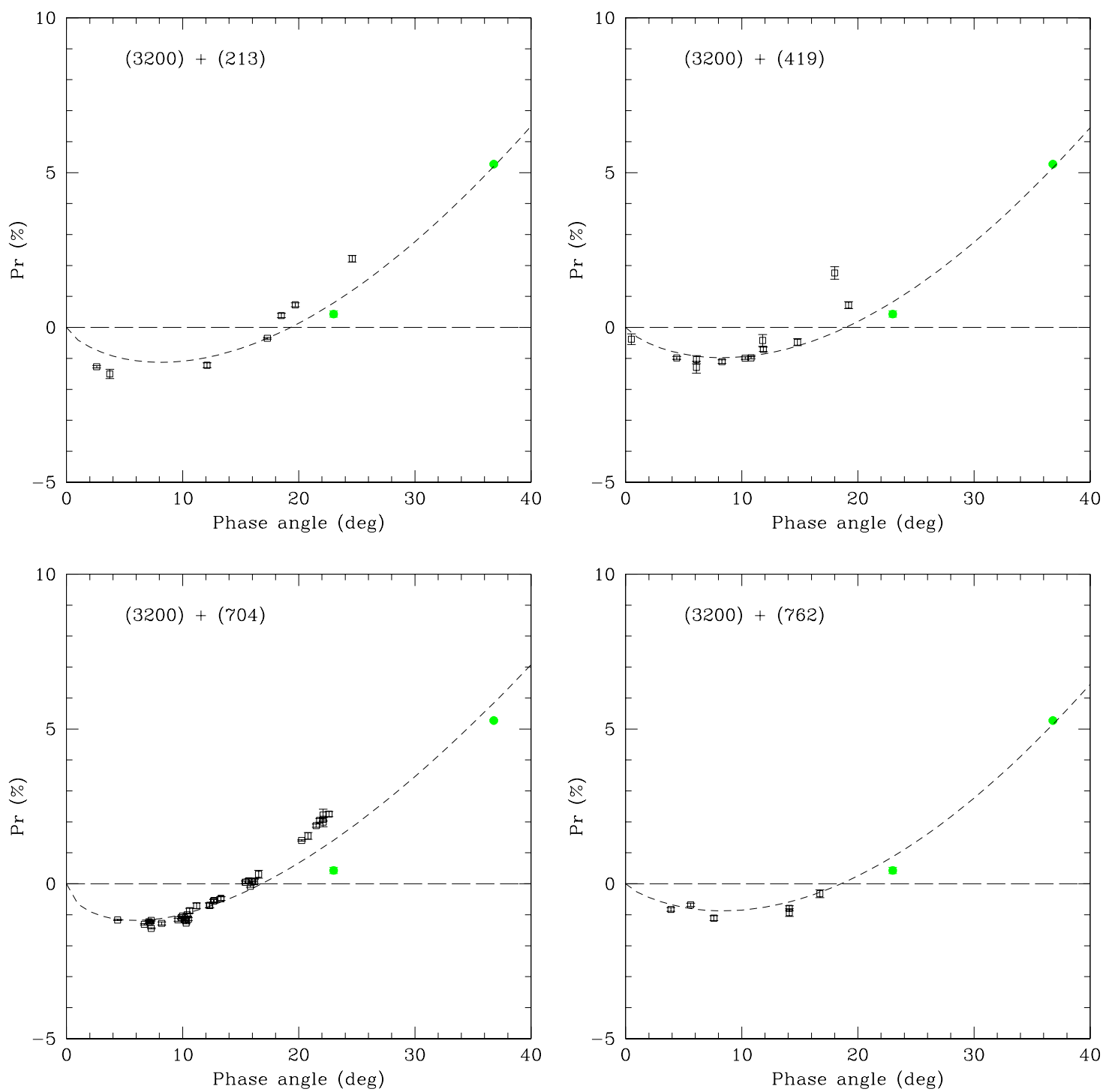

Figure 4. Best-fit phase-polarization curves of the data sets obtained by merging together the (3200) Phaethon V data shown in the left Panel of Fig. 1 and available V data for (213) Lilaea (top left), (419) Aurelia (top right), (704) Interamnia (bottom left) and (762) Pulkova (bottom right). All these asteroids belong to the old F-class. For sake of clarity, the displayed plots cover only the region of phase angle $<40^{\circ}$, as in the right Panel of Fig. 3. Only two measurements of (3200) Phaethon are available in this phase interval, and they are displayed using green symbols. The resulting best-fit curves have been computed in each case using all available data, including also the large number of Phaethon data not shown in the plots.

\section{ACKNOWLEDGEMENTS}

The Torino polarimeter was built at the INAF - Torino Astrophysical Observatory using dedicated funds provided in the framework of the INAF PRIN 2009 program. This work is based on data obtained at the C2PU facility (Calern observing station, Observatoire de la Côte d'Azur, Nice, France) and at the Rozhen National Astronomical Observatory (Bulgaria). GB, ZD and YK gratefully acknowledge observing grant support from the Institute of Astronomy and Rozhen National Astronomical Observatory, Bulgarian Academy of Sciences.

\section{REFERENCES}

Bagnulo S., Cellino A., Sterzik M. F., 2014, MNRAS Letters, 446, L11
Bagnulo S. et al., 2011, JQSRT, 112, 2059

Bagnulo S. et al., 2010, A\&A, 514, A99

Bagnulo S. et al., 2009, PASP, 121, 993

Belskaya I. N. et al., 2017, Icarus, 284, 30

Belskaya I. N. et al., 2005, Icarus, 178, 213

Bus S. J. and Binzel R. P., 2002, Icarus, 158, 146

Bowell E. B. et al., 1992, IAU Circular 5585

Boehnhardt et al., A\&A, 489, 1337

Cellino A., Gil-Hutton R., Belskaya, I. N., 2015, In: Polarimetry of Stars and Planetary Systems (Kolokolova, L., Levasseur-Regourd, A.-C., Hough, J., Eds.), 360, Cambridge University Press, Cambridge, U.K

Cellino A. et al., 2015, MNRAS, 451, 3473

Cellino A. et al., 2016, MNRAS, 455, 2091

Chamberline A. B., Mc Fadden L. A., Schultz R., Schleicher, D. G., Bus S. J., 1996, Icarus, 119, 173

Ciarniello M. et al., 2015, A\&A, 583, A31 


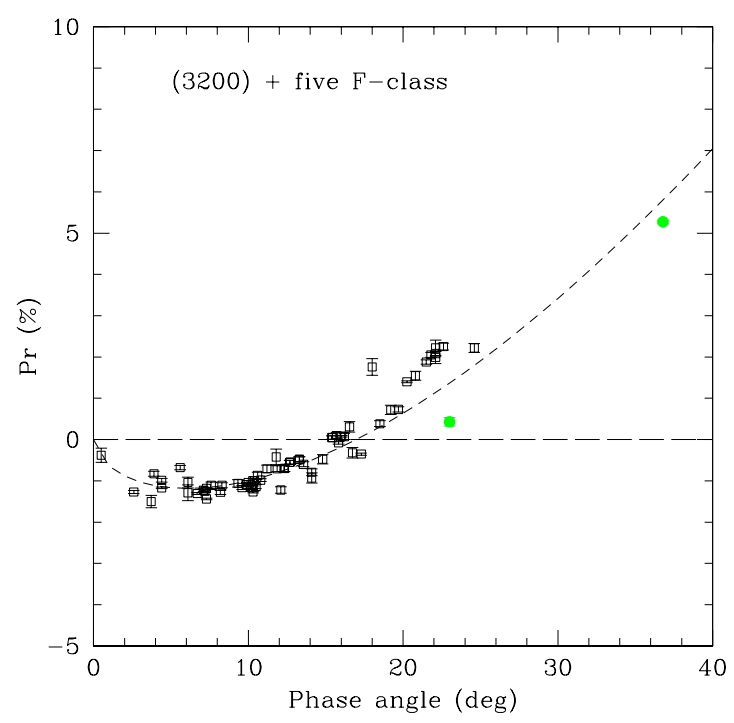

Figure 5. The same as Fig. 4, but here the (3200) Phaethon V data are merged together with all $\mathrm{V}$ data available for the F-class asteroids (142) Polana, (213) Lilaea, (419) Aurelia, (704) Interamnia and (762) Pulkova, shown separately in Figs. 3 and 4.

Cochran A. L. and Barker E. S., 1984, Icarus, 59, 296

De León J., Campins H., Tsiganis K., Morbidelli A., Licandro J., 2010, A\&A, 513, A26

DeMeo F. E. and Binzel R. P., 2008, Icarus, 194, 436

DeMeo F. E., Binzel R. P., Slivan S. M. and Bus S. J., 2009, Icarus, 202, 160

Devogèle M. et al., 2017, MNRAS, 465, 4335

Fernández J., Jewitt D. C., Sheppard S. S., 2005, AJ, 130, 308

Fornasier S. et al., 2006, A\&A, 455, 371

Gustafson B A. S., 1989, A\&A, 225, 533

Gaffey M. J., Bell J. F., and Cruikshank D. P.,, 1989, in Asteroids II (R. P. Binzel, T. Gehrels, M. S. Matthews, Eds.), 98, University of Arizona Press, Tucson

Geake J. E. and Dollfus A., MNRAS, 218, 75

Gil-Hutton R. et al., A\&A, 569, A122

Gradie J. and Tedesco E. F., 1982, Science, 216, 1405

Halliday I., 1988, Icarus, 76, 279

Hanuš J. et al., 2016, A\&A, 592, A34

Hsieh H. H. and Jewitt D., 2006, Science, 312, 561

Jewitt D. and Hsieh H. H., 2006, AJ, 132, 1624

Jockers K. et al., 2000, Kinematika i Fizika Nebesnykh Tel, Suppl., 3, 13

Kolokolova L. and Jockers K., 1997, Planet. Space Sci., 45, 1543

Lumme K. and Muinonen K., 1993, 160 Symposium IAU, Asteroids, Comets, Meteors, 194

Masiero J. R. et al., 2011, ApJ, 749, A104

Milani A., et al., 2014, Icarus, 239, 46

Oliva E., 1997, A\&ASS, 123, 589

Penttila A., Lumme K., Hadamcik E., Levasseur-Regourd A. C., 2005, A\&A, 432, 1081

Pernechele C., Abe L., Bendjoya Ph., Cellino A., Massone G., Tanga P., 2012, Proceedings of the SPIE, 8446, 84462H

Shevchenko V. G. and Tedesco E. F., 2006, Icarus, 184, 211

Tedesco E. F., Noah P. V., Noah M., and Price S. D., 2002, AJ, 123, 1056

Tholen D. J., 1985, IAU Circular 4034

Tholen D. J., 1984, PhD thesis, University of Arizona

\section{APPENDIX A: OBSERVATION DATA}

This paper has been typeset from a $\mathrm{T}_{\mathrm{E}} \mathrm{X} / \mathrm{LAT}_{\mathrm{E}} \mathrm{X}$ file prepared by the author. 
Table A1. Observations of (3200) Phaethon obtained at the Calern and Rozhen observatories. The dates of observation refer to the beginning of the night, and the epochs of observation are given as Modified Julian Date (JD - 2400000.5). The Filter column gives the Johnson-Cousins filter used in each measurement. Phase is the phase angle of Phaethon at the middle of the observation, $P_{\mathrm{r}}$ is the measured polarization, and Observatory corresponds to the observatory were the data were obtained.

\begin{tabular}{|c|c|c|c|c|c|}
\hline Date & MJD & Filter & $\begin{array}{l}\text { Phase } \\
\text { [Deg] }\end{array}$ & $\begin{array}{c}P_{\mathrm{r}} \\
{[\%]}\end{array}$ & Observatory \\
\hline $14 / 12 / 2017$ & 58101.755 & $\mathrm{~V}$ & 36.79 & $5.27 \pm 0.03$ & Calern \\
\hline $14 / 12 / 2017$ & 58101.759 & $\mathrm{R}$ & 36.85 & $5.45 \pm 0.03$ & Calern \\
\hline $14 / 12 / 2017$ & 58101.833 & B & 37.53 & $5.08 \pm 0.03$ & Calern \\
\hline $14 / 12 / 2017$ & 58101.766 & I & 36.92 & $5.71 \pm 0.02$ & Calern \\
\hline $15 / 12 / 2017$ & 58102.696 & $\mathrm{R}$ & 47.48 & $9.89 \pm 0.06$ & Rozhen \\
\hline $15 / 12 / 2017$ & 58102.703 & $\mathrm{R}$ & 47.55 & $9.86 \pm 0.06$ & Rozhen \\
\hline $15 / 12 / 2017$ & 58102.707 & $\mathrm{R}$ & 47.63 & $9.89 \pm 0.07$ & Rozhen \\
\hline $15 / 12 / 2017$ & 58102.712 & $\mathrm{R}$ & 47.69 & $10.01 \pm 0.07$ & Rozhen \\
\hline $15 / 12 / 2017$ & 58102.717 & $\mathrm{R}$ & 47.76 & $10.04 \pm 0.07$ & Rozhen \\
\hline $15 / 12 / 2017$ & 58102.723 & $\mathrm{R}$ & 47.83 & $10.03 \pm 0.07$ & Rozhen \\
\hline $15 / 12 / 2017$ & 58102.729 & $\mathrm{R}$ & 47.91 & $10.07 \pm 0.07$ & Rozhen \\
\hline $15 / 12 / 2017$ & 58102.734 & $\mathrm{R}$ & 47.98 & $10.07 \pm 0.07$ & Rozhen \\
\hline $15 / 12 / 2017$ & 58102.740 & $\mathrm{R}$ & 47.98 & $10.09 \pm 0.06$ & Rozhen \\
\hline $15 / 12 / 2017$ & 58102.745 & $\mathrm{R}$ & 48.12 & $10.10 \pm 0.06$ & Rozhen \\
\hline $15 / 12 / 2017$ & 58102.752 & $\mathrm{R}$ & 48.20 & $10.18 \pm 0.06$ & Rozhen \\
\hline $15 / 12 / 2017$ & 58102.757 & $\mathrm{R}$ & 48.28 & $10.32 \pm 0.07$ & Rozhen \\
\hline $15 / 12 / 2017$ & 58102.763 & $\mathrm{R}$ & 48.36 & $10.24 \pm 0.07$ & Rozhen \\
\hline $15 / 12 / 2017$ & 58102.769 & $\mathrm{R}$ & 48.43 & $10.40 \pm 0.08$ & Rozhen \\
\hline $15 / 12 / 2017$ & 58102.775 & $\mathrm{R}$ & 48.52 & $10.54 \pm 0.08$ & Rozhen \\
\hline $15 / 12 / 2017$ & 58102.781 & $\mathrm{R}$ & 48.59 & $10.46 \pm 0.09$ & Rozhen \\
\hline $15 / 12 / 2017$ & 58102.782 & V & 48.59 & $10.50 \pm 0.04$ & Calern \\
\hline $15 / 12 / 2017$ & 58102.787 & $\mathrm{R}$ & 48.67 & $10.62 \pm 0.10$ & Rozhen \\
\hline $15 / 12 / 2017$ & 58102.822 & V & 49.13 & $10.68 \pm 0.03$ & Calern \\
\hline $15 / 12 / 2017$ & 58102.792 & $\mathrm{R}$ & 48.74 & $10.74 \pm 0.07$ & Rozhen \\
\hline $15 / 12 / 2017$ & 58102.798 & $\mathrm{R}$ & 48.82 & $10.86 \pm 0.06$ & Rozhen \\
\hline $15 / 12 / 2017$ & 58102.804 & $\mathrm{R}$ & 48.89 & $10.81 \pm 0.06$ & Rozhen \\
\hline $15 / 12 / 2017$ & 58102.810 & $\mathrm{R}$ & 47.98 & $10.83 \pm 0.06$ & Rozhen \\
\hline $15 / 12 / 2017$ & 58102.816 & $\mathrm{R}$ & 49.05 & $10.85 \pm 0.06$ & Rozhen \\
\hline $15 / 12 / 2017$ & 58102.822 & $\mathrm{R}$ & 49.12 & $10.86 \pm 0.06$ & Rozhen \\
\hline $15 / 12 / 2017$ & 58102.828 & $\mathrm{R}$ & 49.22 & $10.80 \pm 0.06$ & Rozhen \\
\hline $15 / 12 / 2017$ & 58102.834 & $\mathrm{R}$ & 49.30 & $10.85 \pm 0.06$ & Rozhen \\
\hline $15 / 12 / 2017$ & 58102.840 & $\mathrm{R}$ & 49.36 & $10.75 \pm 0.06$ & Rozhen \\
\hline $15 / 12 / 2017$ & 58102.847 & $\mathrm{R}$ & 49.39 & $10.65 \pm 0.06$ & Rozhen \\
\hline $15 / 12 / 2017$ & 58102.853 & $\mathrm{R}$ & 49.54 & $10.87 \pm 0.06$ & Rozhen \\
\hline $15 / 12 / 2017$ & 58102.866 & V & 49.70 & $10.78 \pm 0.02$ & Calern \\
\hline $15 / 12 / 2017$ & 58102.907 & V & 50.26 & $11.01 \pm 0.03$ & Calern \\
\hline $15 / 12 / 2017$ & 58102.949 & V & 50.82 & $11.69 \pm 0.03$ & Calern \\
\hline $15 / 12 / 2017$ & 58102.786 & $\mathrm{R}$ & 48.66 & $10.78 \pm 0.04$ & Calern \\
\hline $15 / 12 / 2017$ & 58102.827 & $\mathrm{R}$ & 49.19 & $10.90 \pm 0.02$ & Calern \\
\hline $15 / 12 / 2017$ & 58102.861 & $\mathrm{R}$ & 49.77 & $11.03 \pm 0.04$ & Calern \\
\hline $15 / 12 / 2017$ & 58102.912 & $\mathrm{R}$ & 50.32 & $11.34 \pm 0.03$ & Calern \\
\hline $15 / 12 / 2017$ & 58102.953 & $\mathrm{R}$ & 50.87 & $11.90 \pm 0.02$ & Calern \\
\hline $15 / 12 / 2017$ & 58102.793 & I & 48.73 & $11.27 \pm 0.02$ & Calern \\
\hline $15 / 12 / 2017$ & 58102.836 & I & 49.31 & $11.33 \pm 0.02$ & Calern \\
\hline $15 / 12 / 2017$ & 58102.877 & I & 49.85 & $11.55 \pm 0.02$ & Calern \\
\hline $15 / 12 / 2017$ & 58102.918 & I & 50.41 & $11.89 \pm 0.05$ & Calern \\
\hline $15 / 12 / 2017$ & 58102.960 & I & 50.96 & $12.40 \pm 0.02$ & Calern \\
\hline $15 / 12 / 2017$ & 58102.807 & B & 48.91 & $10.19 \pm 0.07$ & Calern \\
\hline $15 / 12 / 2017$ & 58102.850 & B & 49.49 & $10.14 \pm 0.07$ & Calern \\
\hline $15 / 12 / 2017$ & 58102.892 & B & 50.04 & $10.41 \pm 0.04$ & Calern \\
\hline $15 / 12 / 2017$ & 58102.933 & B & 50.59 & $10.95 \pm 0.04$ & Calern \\
\hline $15 / 12 / 2017$ & 58102.976 & B & 51.17 & $11.13 \pm 0.04$ & Calern \\
\hline $16 / 12 / 2017$ & 58103.783 & $\mathrm{~V}$ & 62.57 & $16.93 \pm 0.08$ & Calern \\
\hline $16 / 12 / 2017$ & 58103.812 & V & 62.99 & $17.37 \pm 0.08$ & Calern \\
\hline $16 / 12 / 2017$ & 58103.829 & V & 63.25 & $17.92 \pm 0.08$ & Calern \\
\hline $16 / 12 / 2017$ & 58103.857 & V & 63.66 & $18.37 \pm 0.09$ & Calern \\
\hline $16 / 12 / 2017$ & 58103.876 & V & 63.92 & $18.39 \pm 0.09$ & Calern \\
\hline $16 / 12 / 2017$ & 58103.905 & V & 64.36 & $18.10 \pm 0.09$ & Calern \\
\hline $16 / 12 / 2017$ & 58103.922 & V & 64.62 & $18.30 \pm 0.09$ & Calern \\
\hline $16 / 12 / 2017$ & 58103.960 & V & 65.18 & $18.54 \pm 0.09$ & Calern \\
\hline
\end{tabular}



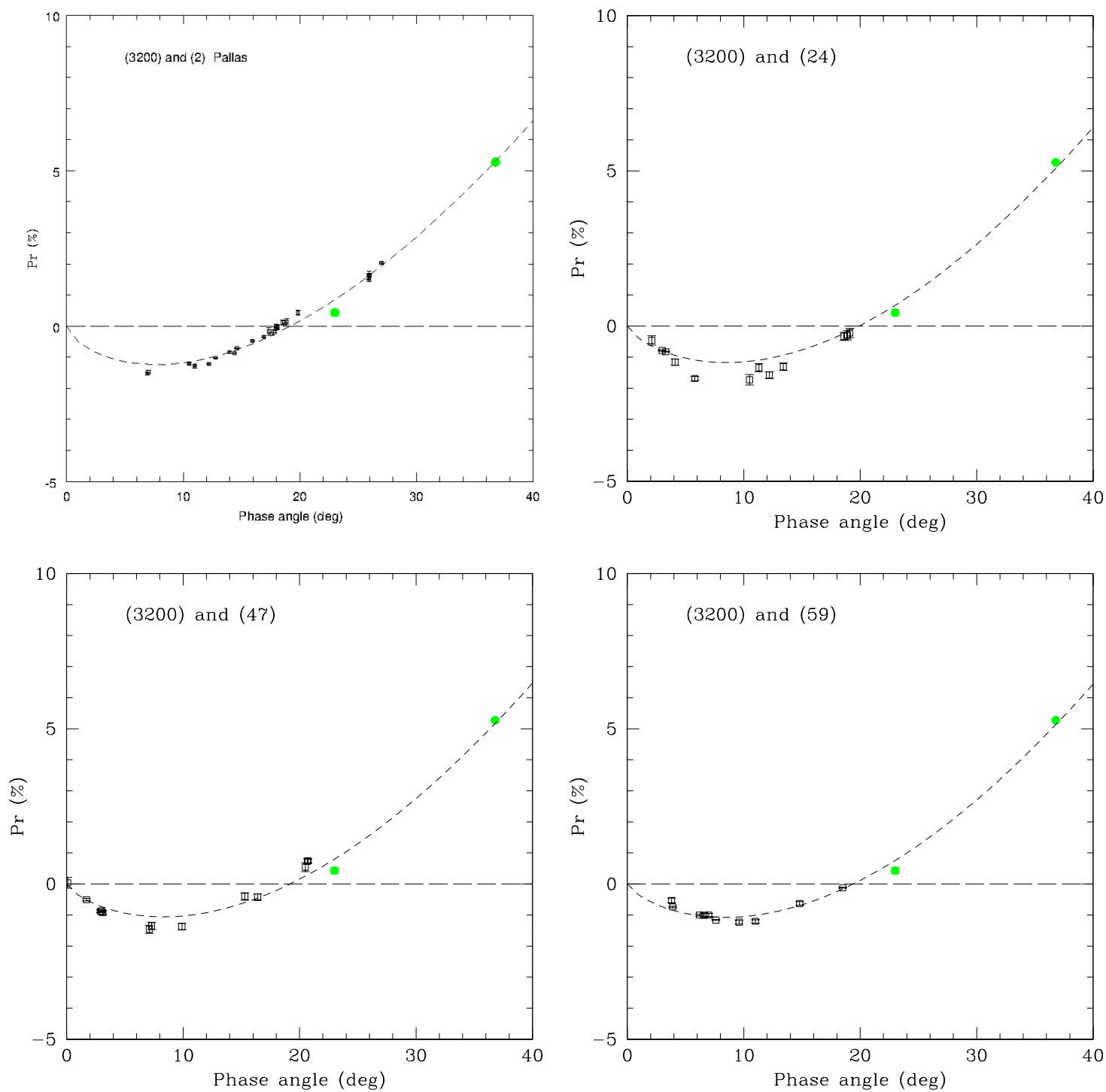

Figure 6. The same as Fig. 4, but here Phaethon polarization data in V light are merged with those for the asteroids (2) Pallas, (23) Themis, (47) Aglaja and (59) Elpis, all belonging to the modern B-class, but not to the old F class. 
Table A1 - continued continued

\begin{tabular}{|c|c|c|c|c|c|}
\hline Date & MJD & Filter & $\begin{array}{l}\text { Phase } \\
\text { [Deg] }\end{array}$ & $\begin{array}{c}P_{\mathrm{r}} \\
{[\%]}\end{array}$ & Observatory \\
\hline $16 / 12 / 2017$ & 58103.789 & $\mathrm{R}$ & 62.66 & $17.27 \pm 0.10$ & Calern \\
\hline $16 / 12 / 2017$ & 58103.818 & $\mathrm{R}$ & 63.08 & $17.87 \pm 0.10$ & Calern \\
\hline $16 / 12 / 2017$ & 58103.835 & $\mathrm{R}$ & 63.33 & $18.46 \pm 0.10$ & Calern \\
\hline $16 / 12 / 2017$ & 58103.862 & $\mathrm{R}$ & 63.74 & $18.78 \pm 0.10$ & Calern \\
\hline $16 / 12 / 2017$ & 58103.881 & $\mathrm{R}$ & 64.03 & $18.56 \pm 0.10$ & Calern \\
\hline $16 / 12 / 2017$ & 58103.910 & $\mathrm{R}$ & 64.44 & $18.55 \pm 0.10$ & Calern \\
\hline $16 / 12 / 2017$ & 58103.931 & $\mathrm{R}$ & 64.75 & $18.55 \pm 0.10$ & Calern \\
\hline $16 / 12 / 2017$ & 58103.967 & $\mathrm{R}$ & 65.29 & $19.12 \pm 0.11$ & Calern \\
\hline $16 / 12 / 2017$ & 58103.795 & I & 62.73 & $18.21 \pm 0.07$ & Calern \\
\hline $16 / 12 / 2017$ & 58103.824 & I & 63.16 & $18.94 \pm 0.08$ & Calern \\
\hline $16 / 12 / 2017$ & 58103.840 & I & 63.41 & $19.37 \pm 0.09$ & Calern \\
\hline $16 / 12 / 2017$ & 58103.869 & I & 63.83 & $19.60 \pm 0.06$ & Calern \\
\hline $16 / 12 / 2017$ & 58103.888 & I & 64.11 & $19.35 \pm 0.07$ & Calern \\
\hline $16 / 12 / 2017$ & 58103.916 & I & 64.52 & $19.65 \pm 0.06$ & Calern \\
\hline $16 / 12 / 2017$ & 58103.939 & I & 64.86 & $19.51 \pm 0.05$ & Calern \\
\hline $16 / 12 / 2017$ & 58103.804 & B & 62.86 & $16.66 \pm 0.05$ & Calern \\
\hline $16 / 12 / 2017$ & 58103.849 & B & 63.51 & $17.85 \pm 0.07$ & Calern \\
\hline $16 / 12 / 2017$ & 58103.897 & B & 64.21 & $17.30 \pm 0.06$ & Calern \\
\hline $16 / 12 / 2017$ & 58103.950 & B & 65.00 & $17.65 \pm 0.09$ & Calern \\
\hline $17 / 12 / 2017$ & 58104.780 & $\mathrm{R}$ & 77.12 & $26.65 \pm 0.12$ & Calern \\
\hline $17 / 12 / 2017$ & 58104.822 & $\mathrm{R}$ & 77.72 & $25.04 \pm 0.23$ & Calern \\
\hline $17 / 12 / 2017$ & 58104.865 & $\mathrm{R}$ & 78.34 & $26.42 \pm 0.10$ & Calern \\
\hline $17 / 12 / 2017$ & 58104.882 & $\mathrm{R}$ & 78.57 & $27.25 \pm 0.10$ & Calern \\
\hline $17 / 12 / 2017$ & 58104.900 & $\mathrm{R}$ & 78.82 & $27.95 \pm 0.11$ & Calern \\
\hline $17 / 12 / 2017$ & 58104.802 & B & 77.42 & $24.555 \pm 0.12$ & Calern \\
\hline $17 / 12 / 2017$ & 58104.844 & B & 78.03 & $24.147 \pm 0.13$ & Calern \\
\hline $17 / 12 / 2017$ & 58104.787 & I & 77.21 & $27.172 \pm 0.08$ & Calern \\
\hline $17 / 12 / 2017$ & 58104.829 & I & 77.82 & $26.981 \pm 0.09$ & Calern \\
\hline $17 / 12 / 2017$ & 58104.871 & I & 78.42 & $27.675 \pm 0.09$ & Calern \\
\hline $17 / 12 / 2017$ & 58104.889 & I & 78.66 & $28.412 \pm 0.07$ & Calern \\
\hline $17 / 12 / 2017$ & 58104.775 & V & 77.04 & $25.942 \pm 0.09$ & Calern \\
\hline $17 / 12 / 2017$ & 58104.816 & V & 77.63 & $25.563 \pm 0.09$ & Calern \\
\hline $17 / 12 / 2017$ & 58104.861 & V & 78.27 & $25.535 \pm 0.09$ & Calern \\
\hline $17 / 12 / 2017$ & 58104.878 & V & 78.51 & $26.39 \pm 0.09$ & Calern \\
\hline $17 / 12 / 2017$ & 58104.895 & V & 78.76 & $27.201 \pm 0.11$ & Calern \\
\hline $19 / 12 / 2017$ & 58106.807 & V & 101.57 & $36.229 \pm 0.16$ & Calern \\
\hline $19 / 12 / 2017$ & 58106.829 & V & 101.78 & $38.402 \pm 0.09$ & Calern \\
\hline $19 / 12 / 2017$ & 58106.833 & V & 101.81 & $38.539 \pm 0.09$ & Calern \\
\hline $19 / 12 / 2017$ & 58106.836 & V & 101.84 & $38.684 \pm 0.09$ & Calern \\
\hline $19 / 12 / 2017$ & 58106.838 & V & 101.87 & $38.587 \pm 0.10$ & Calern \\
\hline $19 / 12 / 2017$ & 58106.841 & V & 101.90 & $38.675 \pm 0.09$ & Calern \\
\hline $19 / 12 / 2017$ & 58106.846 & V & 101.94 & $39.216 \pm 0.10$ & Calern \\
\hline $20 / 12 / 2017$ & 58107.701 & $\mathrm{R}$ & 109.25 & $41.17 \pm 0.06$ & Rozhen \\
\hline $21 / 12 / 2017$ & 58108.718 & $\mathrm{R}$ & 116.25 & $43.50 \pm 0.10$ & Rozhen \\
\hline
\end{tabular}




\section{Devogèle et al.}

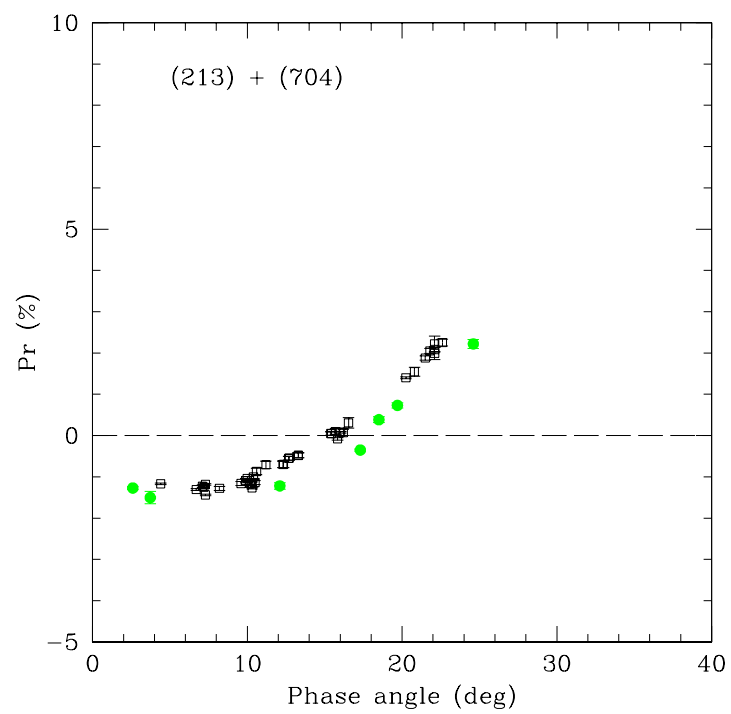

Figure 7. Available phase-polarization measurements in $\mathrm{V}$ colour for the two F-class asteroids (213) Lilaea (green symbols) and (704) Interamnia (black symbols). There are differences between the two objects, but both exhibit an inversion angle of polarization, between about $16^{\circ}$ and $18^{\circ}$, which is low in comparison to the vast majority of asteroids belonging to other taxonomic classes. 